\title{
L-ORTHOGONAL SIGNAL TRANSMISSION AND DETECTION
}

BY W. C. LINDSEY and M. K. SIMON

Summary. This paper investigates the detailed capabilities and performance characterization of systems that employ L-orthogonal signaling techniques. L-orthogonal signals represent a unified set of signals wherein the polyphase and orthogonal (bi-orthogonal) signal sets are included as special cases. This fact is important since orthogonal (bi-orthogonal) and polyphase signaling sets represent opposing forces as far as tradeoffs between error probability, energy -to-noise ratio, and bandwidth requirements are concerned. Bounds on the performance of the optimum receiver and the performance of various suboptimum (practical) receiver structures are given. Coherent and differentially coherent detection of differentially encoded signals are also pursued. Various comparisons and tradeoffs are made by means of numerical evaluation of the error probability expressions.

Introduction. With the high communication traffic density predicted for future data transmission systems, it is important to investigate and compare all possible modulation techniques that are practical for such applications. In particular, one is interested in the tradeoff between the bandwidth required to transmit the given signaling scheme and the error probability performance of the associated receiver used for data detection.

Because of its desirable bandwidth properties, considerable attention has been focussed on multiple phase-shift-keying (MPSK), i. e., the use of polyphase signals for data transmission, as a suitable modulation technique. In particular, quadriphase-shift-keying (QPSK) appears to be attractive over binary PSK since it offers a $3-\mathrm{dB}$ saving in bandwidth at no loss in system bit error probability performance. Higher order phase-shiftkeyed modulation formats (Ref. 1) could be employed to give further reduction in bandwidth occupancy; however, the accompanying increase in error probability for a given signal-to-noise ratio per bit now becomes significant. Such a loss in performance would be incurred regardless of whether coherent or differentially coherent detection is employed at the receiver. The performance of these types of receivers using differential encoding of the data for ambiguity resolution is given in Ref. 2. Also, the capabilities of combined phase-shift-keyed and amplitude modulation have been investigated (Ref. 3). 
Recognizing that polyphase signaling is basically an uncoded signal transmission scheme, i.e., multilevel digital phase modulation of a known carrier, one naturally asks about the performance benefits gained through coding at the expense, of course, of an increase in bandwidth. In this connection, the error probability performance of signaling sets based upon orthogonal and bi-orthogonal codes has been extensively studied in the literature (Refs. 4, 5).

Since orthogonal (bi-orthogonal) and polyphase signaling sets represent opposing forces insofar as tradeoffs between performance and bandwidth are concerned, one is motivated to study the performance capability of Lorthogonal signals (Ref. 6) whose properties enable them to bridge the merits of both of the former signaling schemes. Hence, a study of Lorthogonal signal performance represents a unified approach wherein the performance of polyphase and orthogonal (bi-orthogonal) signal sets stand out as the extreme special cases. After a brief discussion of the Lorthogonal signal model and its associated bandwidth properties, we begin our discussion of system performance by presenting tight upper and lower bounds on the error probability performance of the maximum-likelihood receiver of L-orthogonal signals suggested by Reed and Scholtz (Ref. 6). Because it is impractical to implement the maximum-likelihood receiver, the performance (assuming perfect reference signals) of a practical suboptimum receiver (Ref. 7) is presented next and compared with the bounds derived for the optimum receiver. Also included in our performance study of the suboptimum receiver is its effectiveness in terms of coherent and differentially coherent detection of differentially encoded signals. Finally, we conclude with a study of the error probability performance of the suboptimum receiver in the presence of noisy reference signals. The results presented in this paper are of interest to communication system engineers faced with the problems of system design, comparison, and planning of high capacity data transmission systems.

Signal Representation and Bandwidth Considerations. An L-orthogonal signal set consists of $\mathrm{N}=\mathrm{ML}$ equal energy $(\mathrm{E}=\mathrm{ST})$, equal duration ( $\mathrm{T}$ ) signals $\left\{s_{i}(t) ; i=1,2, \ldots, N\right\}$ that can be subdivided into $M$ subsets of $L$ signals each with the following properties:

(1) Any pair of signals not in the same subset are orthogonal.

(2) The $i^{\text {th }}$ and $j^{\text {th }}$ signals of the same subset have a normalized correlation (inner product).

$$
\lambda_{i j}=\frac{1}{S T} \int_{0}^{T} s_{i}(t) s_{j}(t) d t=\cos [2 \pi|i-j| / L] ; i, j=1,2, \ldots, L
$$

Thus, the $N \times N$ correlation matrix $\Delta_{L}$ O for the entire set of $N$ signals can be partitioned so that it contains $\mathrm{M}(\tilde{L} \times \mathrm{L})$ submatrices along its principal diagonal each corresponding to the correlation matrix for L polyphase signals, and all other elements zero. 
The construction of signal sets that possess these correlation properties has been suggested by several authors (Refs. 6, 7). In all cases, the signals can be expressed in the form

$$
\begin{aligned}
\mathrm{s}_{\mathrm{i}}(\mathrm{t}) & =\sqrt{\mathrm{S}}\left[\mathrm{x}_{\mathrm{m}}(\mathrm{t}) \cos \theta_{\ell}+\mathrm{y}_{\mathrm{m}}(\mathrm{t}) \sin \theta_{\ell}\right] ; 0 \leq \mathrm{t} \leq \mathrm{T} \\
\mathrm{m} & =1,2, \ldots \ldots, \mathrm{M} \\
\ell & =1,2, \ldots \ldots, L \\
\mathrm{i} & =(\mathrm{m}-1) L+\ell
\end{aligned}
$$

where $\theta_{\ell}=2(\ell-1) \pi / L$,

$$
\frac{1}{T} \int_{0}^{T} x_{k}(t) x_{n}(t) d t=\frac{1}{T} \int_{0}^{T} y_{k}(t) y_{n}(t) d t= \begin{cases}1 & k=n \\ 0 & k \neq n\end{cases}
$$

and

$$
\frac{1}{T} \int_{0}^{T} x_{k}(t) y_{n}(t) d t=0 \quad \text { all } k \text { and } n
$$

In forming the cross-correlation between $s_{i}(t)$ and $s_{j}(t)$ where $i=\left(m_{1}-1\right)$ $\times L+\ell_{1}, j=\left(m_{2}-1\right) L+\ell_{2}\left(m_{1} \neq m_{2}\right)$, the coefficient of the cross-correlation between $\mathrm{x}_{\mathrm{m}_{1}}(\mathrm{t})$ and $\mathrm{y}_{\mathrm{m}_{2}}(\mathrm{t})$, or $\mathrm{x}_{\mathrm{m}_{2}}(\mathrm{t})$ and $\mathrm{y}_{\mathrm{m}_{1}}(\mathrm{t})$ depends on $\sin \left(\theta_{\ell_{1}}-\theta_{\ell_{2}}\right)$ and $\sin \left(\theta_{\ell_{1}}+\theta_{\ell_{2}}\right.$ ). For $L=1$, or $L=2$ (corresponding to the cases of orthogonal and bi-orthogonal signals respectively), $\theta_{\ell_{1}}-\theta_{\ell_{2}}$ and $\theta_{\ell_{1}}+\theta_{\ell_{2}}$ are either zero or an integer multiple of $\pi$. Hence, regardless of the value of the cross-correlation between $\mathrm{x}_{\mathrm{m}_{1}}(\mathrm{t})$ and $\mathrm{y}_{\mathrm{m}_{2}}(\mathrm{t})$, or $\mathrm{x}_{\mathrm{m}_{2}}(\mathrm{t})$ and $\mathrm{y}_{\mathrm{m}_{1}}(\mathrm{t})$, the orthogonality of $s_{i}(t)$ and $s_{j}(t)$ is satisfied since $\sin \left(\theta_{\ell_{1}}-\theta_{\ell_{2}}\right)$ and $\sin \left(\theta_{\ell_{1}}+\theta_{\ell_{2}}\right)$ are both equal to zero. Thus, we conclude that the orthogonality relationship of (4) for $k \neq n$ is required only for $L>2$.

In the literature, one can find many practical methods for generating the orthogonal signal sets $\left\{\mathrm{x}_{\mathrm{m}}(\mathrm{t})\right\}$ and $\left\{\mathrm{y}_{\mathrm{m}}(\mathrm{t})\right\}$. In terms of demonstrating how the bandwidth of the L-orthogonal signal set changes as $L$ and $M$ are varied at fixed $N$, it is convenient to use the representation of $\left\{x_{m}(t)\right\}$ and $\left\{y_{m}(t)\right\}$ corresponding to a set of $M$ sinusoidal tones typical of those used in MFSK signaling systems, i.e.,

$$
\begin{array}{ll}
x_{m}(t)=\sqrt{2} \sin \left[\frac{\pi(k+m) t}{T}\right] & L=1,2 \\
y_{m}(t)=\sqrt{2} \cos \left[\frac{\pi(k+m) t}{T}\right] & m=1,2, \ldots, M
\end{array}
$$


and

$$
\begin{array}{ll}
x_{m}(t)=\sqrt{2} \sin \left[\frac{\pi(k+2 m) t}{T}\right] & L>2 \\
y_{m}(t)=\sqrt{2} \cos \left[\frac{\pi(k+2 m) t}{T}\right] & m=1,2, \ldots, M
\end{array}
$$

where $\mathrm{k}$ is an arbitrary integer. Notice that for $\mathrm{L}=1,2$, the signal frequencies are separated by $1 / 2 \mathrm{~T} \mathrm{~Hz}$ whereas for $\mathrm{L}>2$, they must be separated by $1 / \mathrm{T} \mathrm{Hz}$ to insure the mutual orthogonality as required by (4). Substituting (5) into (2), we get

$$
s_{i}(t)=\left\{\begin{array}{rl}
\sqrt{2 S} \sin \left[\frac{\pi(k+m) t}{T}+\theta_{\ell}\right] ; L=1,2 & m=1,2, \ldots, M \\
\ell & =1,2, \ldots, L \\
\sqrt{2 S} \sin \left[\frac{\pi(k+2 m) t}{T}+\theta_{\ell}\right] ; L>2 & i=(m-1) L+\ell
\end{array}\right.
$$

When $M=N(L=1)$, we transmit in each $T-$ second interval a sine wave having one of $\mathrm{N}$ frequencies and zero phase. This signaling scheme corresponds to the well-known case of phase-coherent orthogonal (MFSK) signaling and has an effective bandwidth $\mathrm{W}_{\mathrm{e}}=\mathrm{N} / 2 \mathrm{~T}$. When $\mathrm{M}=\mathrm{N} / 2(\mathrm{~L}=2)$, we transmit in each $\mathrm{T}$-second interval one of $\mathrm{N} / 2 \mathrm{frequencies}$ at a phase of either zero or $\pi$ radians. The effective bandwidth for this bi-orthogonal signaling technique is $\mathrm{W}_{\mathrm{e}}=\mathrm{N} / 4 \mathrm{~T}$, which is one half of the bandwidth of the orthogonal signaling case. If $\mathrm{M}$ is now reduced by another factor of 2 , we have a situation wherein one of $\mathrm{N} / 4$ frequencies at a phase of zero, $\pi / 2, \pi$, or $3 \pi / 2$ radians is transmitted in each $\mathrm{T}$-second interval. Since the frequency separation has now increased by a factor of 2 [see (6)], the effective bandwidth is $\mathrm{W}_{\mathrm{e}}=\mathrm{N} / 4 \mathrm{~T}$ and is thus the same as for $\mathrm{L}=2$. This is not surprising when we realize that a quadriphase-modulated sinusoid can be represented as two orthogonal biphase-modulated sinusoids, and hence the signal representations for $L=2$ and $L=4$ are equivalent and as we shall see later yield identical performance. From this point, further reduction in $M$ results in further reduction in $W_{e}$ and, in general (for $L>2$ ), $W_{e}=M / T$. When $M=1(L=N)$, we have the case of polyphase signaling that occupies the least bandwidth.

Error Probability Bounds for the Maximum-Likelihood Receiver. The optimum (maximum-likelihood) receiver for transmission of L-orthogonal signals over an additive Gaussian noise channel is a bank of $\mathrm{N}$ matched filters matched to the possible transmitted signals. The error probability performance of this receiver has been studied by Reed and Scholtz (Ref. 6). For $L>2$, the probability of correct detection is given by

$$
P_{C}(N)=\iint_{B} \frac{\exp \left[-\left(x_{1}^{2}+x_{2}^{2}\right)\right]}{\pi}\left\{\iint_{A\left(x_{1}\right)} \frac{\exp \left[-\left(y_{1}^{2}+y_{2}^{2}\right)\right]}{\pi} d y_{1} d y_{2}\right\}^{M-1} d x_{1} d x_{2}
$$

For $\mathrm{L}=1$ and $\mathrm{L}=2$, we have the well known results for orthogonal and bi-orthogonal signaling respectively. 
where $A\left(x_{1}\right)$ and $B$ are regions of integration (see Fig. 1). In particular, if $R_{d} \triangleq S T / N_{0}$, then $B$ is the set of points $\left(x_{1}, x_{2}\right)$ in the wedge bounded by the straight lines

$$
\begin{aligned}
& x_{2}=\left(x_{1}+\sqrt{R_{d}}\right) \tan \frac{\pi}{L} \\
& x_{2}=\left(-x_{1}+\sqrt{R_{d}}\right) \tan \frac{\pi}{L}
\end{aligned}
$$

and $\mathrm{A}\left(\mathrm{x}_{1}\right)$ is the set of points within an L-sided regular polygon circumscribed on a circle of radius $x_{1}+\sqrt{R_{d}}$. Substituting (8) in (7) and making the change of variables $u_{1}=x_{1}+\sqrt{R_{d}}$, we have:

$$
\begin{aligned}
P_{c}(N)= & \frac{2}{\pi} \int_{0}^{\infty} \exp \left[-\left(\mathrm{u}_{1}-\mathrm{R}_{\mathrm{d}}\right)^{2}\right] \\
& \times\left[\int_{0}^{\mathrm{u}_{1} \tan \pi / \mathrm{L}} \exp \left(-\mathrm{x}_{2}^{2}\right) \mathrm{dx_{2 }}\right]\left\{\int_{\mathrm{A}_{\left(\mathrm{u}_{1}\right)}} \frac{\exp \left[-\left(\mathrm{y}_{1}^{2}+\mathrm{y}_{2}^{2}\right)\right]}{\pi} \mathrm{dy}_{1} \mathrm{dy}_{2}\right\}^{\mathrm{M}-1} \mathrm{du} \mathrm{u}_{1}
\end{aligned}
$$

Since any L-sided regular polygon can be subdivided into $L$ equal area triangles, then from the symmetry of the double integral on $A\left(u_{1}\right)$, we get*

$$
\begin{aligned}
P_{c}(N)= & \frac{2}{\pi} \int_{0}^{\infty} \exp \left[-\left(u_{1}-R_{d}\right)^{2}\right]\left[\int_{0}^{u_{1} \tan \pi / L} \exp \left(-x_{2}^{2}\right) d x_{2}\right] \\
& \times\left\{L \int_{0}^{u_{1} l} \int_{-y_{1}}^{y_{1} \tan \pi / L} \frac{\exp \left[-\left(y_{1}^{2}+y_{2}^{2}\right)\right]_{d}}{\pi} d_{1} d_{2}\right\}^{M-1} d u_{1} \\
= & \frac{1}{\sqrt{\pi}} \int_{0}^{\infty} \exp \left[-\left(u_{1}-R_{d}\right)^{2}\right] \operatorname{erf}\left(u_{1} \tan \frac{\pi}{L}\right) \\
& \times\left[\frac{L}{\sqrt{\pi}} \int_{0}^{u_{1}} \exp \left(-y_{1}^{2}\right) \operatorname{erf}\left(y_{1} \tan \frac{\pi}{L}\right) d_{1}\right]_{1}^{M-1} d_{1}
\end{aligned}
$$

*We define the error function erf(x) and complementary error function $\operatorname{erfc}(x)$ by

$$
\begin{aligned}
& \operatorname{erf}(x)=\frac{2}{\sqrt{\pi}} \int_{0}^{x} \exp \left(-y^{2}\right) d y \\
& \operatorname{erfc}(x)=1-\operatorname{erf}(x)
\end{aligned}
$$


The signal set for $\mathrm{L}=4$ is equivalent to a bi-orthogonal signal set and hence (10) reduces to

$$
P_{c}(N)=\int_{-\sqrt{R_{d}}}^{\infty} \frac{\exp \left(-x_{1}^{2}\right)}{\sqrt{\pi}}\left[1-\operatorname{erfc}\left(x_{1}+R_{d}\right)\right]^{N / 2-1} d x_{1}
$$

When $M=1$, we merely have a single set of polyphase signals and by inspection (10) reduces to

$$
P_{c}(N)=\frac{1}{\sqrt{\pi}} \int_{0}^{\infty} \exp \left[-\left(u_{1}-R_{d}\right)^{2}\right] \operatorname{erf}\left(u_{1} \tan \frac{\pi}{N}\right) d u_{1}
$$

For other combinations of $\mathrm{M}$ and $\mathrm{L}$, the integrals in (10) must be evaluated by numerical integration on a general purpose digital computer.

For $\mathrm{L}>2$, upper and lower bounds on the probability of word error $P_{E}(N)=1-P_{C}(N)$ may be readily calculated by circular approximation to the area of integration $A\left(u_{1}\right)$. In particular, an upper bound on $P_{E}(N)$ [lower bound on $P_{C}(N)$ ] is obtained by approximating $A\left(u_{1}\right)$ by its inscribed circle of radius $u_{1}$. Similarly, a lower bound on $P_{E}(N)$ may be calculated by approximating $A\left(u_{1}\right)$ by its circumscribed circle of radius $u_{1} \sec (\pi / L)$. The results are

$$
\begin{aligned}
P_{E}(N) \leq P_{E_{u}}(N)= & \left.P_{E}(L)\right|_{\text {MPSK }} \\
& -\sum_{k=1}^{M-1} \frac{(-1)^{k}}{(1+k)} M-1 C_{k} \exp \left(-\frac{k R d}{1+k}\right)\left[\frac{\theta}{\pi}+\frac{1}{2} \operatorname{erf}\left(\sqrt{\frac{R_{d}}{1+k}} \sin \theta\right)\right. \\
& \left.+\frac{1}{\sqrt{\pi}} \int_{0}^{\sqrt{R_{d} /(1+k)} \sin \theta} \exp \left(-y^{2}\right) \operatorname{erf}(y \cot \theta) d y\right] \\
\theta= & \tan ^{-1}\left[\frac{\tan \frac{\pi}{L}}{\sqrt{1+k}}\right]
\end{aligned}
$$




$$
\begin{aligned}
P_{E}(N) \geq P_{E_{\ell}}(N)= & \left.P_{E}(L)\right|_{M P S K} \\
& -\sum_{k=1}^{M-1} \frac{(-1)^{k}}{1+k^{\prime}} M-1 C_{k} \exp \left(-\frac{k^{\prime} R_{d}}{1+k^{1}}\right)\left[\frac{\theta^{\prime}}{\pi}+\frac{1}{2} \operatorname{erf}\left(\sqrt{\frac{R_{d}}{1+k}} \sin \theta^{\prime}\right)\right. \\
& \left.+\frac{1}{\sqrt{\pi}} \int_{0}^{\sqrt{R_{d} /\left(1+k^{\prime}\right)} \sin \theta^{\prime}} \exp \left(-y^{2}\right) \operatorname{erf}\left(y \cot \theta^{\prime}\right) d y\right] \\
\theta^{\prime}= & \tan ^{-1}\left[\frac{\tan \frac{\pi}{L}}{1+k^{\prime}}\right]
\end{aligned}
$$

where

$$
M-1 C_{k} \triangleq \frac{(M-1) !}{k !(M-1-k) !}
$$

and

$$
\left.\mathrm{P}_{\mathrm{E}}(\mathrm{L})\right|_{\text {MPSK }} \triangleq 1-\left.\mathrm{P}_{\mathrm{C}}(L)\right|_{\text {MPSK }}
$$

with $\left.P_{C}(L)\right|_{\text {MPSK }}$ obtained from (12) with $N$ replaced by $L$.

We note from (13) and (14) that for $M=1$, the two bounds become equal and give the exact performance; that is, $P_{E}(L) \mid$ MPSK . Also, for large L ( small $\mathrm{M}$ ), the bounds are very tight since both the inscribed and circumscribed circles are good approximations to the L-sided polygon. Fig. 2 illustrates the upper and lower bounds of (13) and (14) respectively as functions of $\mathrm{R}_{\mathrm{b}}=\mathrm{R}_{\mathrm{d}} / \log _{2} \mathrm{~N}$ in $\mathrm{db}$ for $\mathrm{N}=64$ and various combinations of $\mathrm{M}$ and $\mathrm{L}$.

Ideal Word Error Probability Performance of a Suboptimum Receiver. As already discussed, optimum detection of the set of signals in (2) requires a correlation receiver with $\mathrm{N}$ correlators and yields the performance as given by (7). When $\mathrm{N}$ becomes large, the number of correlators required to implement this receiver becomes prohibitive. Hence, we investigate a suboptimum receiver that requires only $2 \mathrm{M}$ correlators and whose error probability performance is quite close to that of the optimum receiver.

The suboptimum receiver illustrated in Fig. 3 uses a noncoherent detector to determine the most probable index $\mathrm{m}$ characterizing the received signal [see (2)] and a coherent or differentially coherent detector to measure the phase $\theta_{\ell}$, or equivalently determine the index $\ell$. The probability, $\left.P_{c}(M)\right|_{N O N}$, of correctly determining the index $m$ from a total of $M$ choices is given by (Ref. 8 )

$$
\left.P_{c}(M)\right|_{N O N}=\sum_{k=0}^{M-1} \frac{(-1)^{k}}{1+k} M_{-1} C_{k} \exp \left(-\frac{k R}{1+k}\right)
$$


The conditional probability, $\left.\mathrm{P}_{\mathrm{c}}(\mathrm{L})\right|_{\theta}$, of correctly determining the phase $\theta_{\ell}$ from a total of $L$ choices, given that $m$ has been correctly chosen, depends on the structure of the phase estimator in Fig. 3. Before examining specific phase estimator configurations, we continue with our characterization of the general form of the suboptimum receiver as given in Fig. 3. Since the index i [see (2)] will be correct only if $m$ and $\ell$ are correctly chosen, then from Bayes' rule, the probability of correct word detection for this receiver is

$$
P_{c}(N)=\left[\left.P_{c}(M)\right|_{N O N}\right]\left[\left.P_{c}(L)\right|_{\theta}\right]
$$

or equivalently, the probability of word error is given by

$$
\mathrm{P}_{E}(\mathrm{~N})=\left.\mathrm{P}_{E}(\mathrm{M})\right|_{N O N}+\left.\mathrm{P}_{E}^{(L)}\right|_{\theta}-\left[\left.\mathrm{P}_{E}^{(M)}\right|_{N O N}\right]\left[\left.\mathrm{P}_{E}^{(L)}\right|_{\theta}\right]
$$

Figure 4 illustrates three possible phase estimator configurations corresponding respectively to the cases of (1) MPSK (maximumlikelihood detection of $\left.\theta_{\ell}\right),(2)$ coherent MPSK with differential encoding of $\theta_{\ell}$, and (3) differentially coherent MPSK (MDPSK) with differential encoding of $\theta_{\ell}$. The conditional error probability performance of each of these configurations is investigated in Refs. 1, 2, and 9 respectively, and is summarized below.

(1) MPSK (maximum-likelihood detection of $\theta_{\ell}$ )

$$
\left.P_{E}(L)\right|_{\theta}=1-\left.P_{c}(L)\right|_{\text {MPSK }}
$$

where $\left.P_{c}(L)\right|_{\text {MPSK }}$ is obtained from (12) with $N$ replaced by $L$.

(2) Coherent MPSK with differential encoding of $\theta_{\ell}$

$$
\left.P_{E}(L)\right|_{\theta}=1-\left[\left.P_{c}(L)\right|_{M P S K}\right]^{2}-\sum_{k=1}^{L-1} P_{E_{k}}^{2}(L)
$$

where

$$
\begin{aligned}
\mathrm{P}_{E_{k}}(L) & =\frac{1}{\pi} \int_{0}^{\infty}\left\{\exp \left[-\left(u-\sqrt{R_{d}}\right)^{2}\right] \int_{u \tan \frac{(2 k-1)}{L} \pi}^{u \tan \frac{(2 k+1)}{L} \pi} \exp \left(-v^{2}\right) d v\right\} d u \\
& =\frac{1}{L}+\frac{1}{4} \operatorname{erf}\left[\sqrt{R_{d}} \sin \frac{(2 k+1)}{L} \pi\right]-\frac{1}{4} \operatorname{erf}\left[\sqrt{R_{d}} \sin \frac{(2 k-1)}{L} \pi\right]
\end{aligned}
$$




$$
\begin{aligned}
& +\frac{1}{2 \sqrt{\pi}} \int_{0}^{\sqrt{R_{d}} \sin \frac{(2 k+1)}{L} \pi} e^{-y^{2}} \operatorname{erf}\left[y \cot \frac{(2 k+1)}{L} \pi\right] d y \\
& -\frac{1}{2 \sqrt{\pi}} \int_{0}^{\sqrt{R_{d}} \sin \frac{(2 k-1)}{L} \pi} e^{-y^{2}} \operatorname{erf}\left[y \cot \frac{(2 k-1)}{L} \pi\right] d y
\end{aligned}
$$

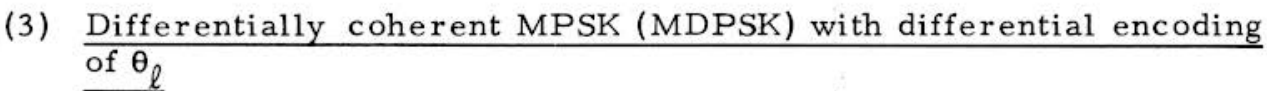

$$
\left.P_{E}(L)\right|_{\theta}=1-\int_{-\pi / L}^{\pi / L} p_{\Psi}(\psi) d \psi
$$

where

$$
\begin{aligned}
\mathrm{p}_{\Psi}(\psi)= & \frac{1}{2 \pi} \int_{0}^{\pi / 2}(\sin \alpha)\left[1+\mathrm{R}_{\mathrm{d}}(1+\cos \psi \sin \alpha)\right] \\
& \times \exp \left[-\mathrm{R}_{\mathrm{d}}(1-\cos \psi \sin \alpha)\right] \mathrm{d} \alpha
\end{aligned}
$$

Substituting (18), (19), or (21) in (17), we have the error probability performance of the suboptimum receiver whose phase estimator in the $\mathrm{m}^{\text {th }}$ branch $\mathrm{m}=1,2, \ldots . M$ is respectively that of Fig. $4 \mathrm{a}, 4 \mathrm{~b}$, and $4 \mathrm{c}$. The corresponding error probability performances are illustrated in Figs. 2, 5, and 6 for $N=64$ and various combinations of $M$ and $L$ whose values are powers of 2 .

Error Probability Performance of a Suboptimum Receiver with Noisy Reference Signals. As in any practical receiver employing carrier tracking loops for establishing coherent reference signals, the phase jitter associated with these reference signals caused by the additive Gaussian noise perturbing the loop produces a degradation of error probability performance. As always, the first step in solving the so-called "noisy reference problem" is to establish an expression for the probability of error conditioned on a given noisy reference phase error. For the suboptimum receiver of Fig. 3, we need only be concerned with establishing the conditional probability of error associated with the phase estimation since the magnitude estimation is noncoherent and hence independent of the noisy reference phase error.

A generalization of (17) yields

$$
P_{E}(\phi)=\left.P_{E}(M)\right|_{N O N}+P_{E}(L ; \phi)-\left[\left.P_{E}(M)\right|_{N O N}\right]\left[P_{E}(L ; \phi)\right]
$$

as the conditional word error probability of the suboptimum receiver with $\mathrm{P}_{\mathrm{E}}(\mathrm{L} ; \phi)$, the conditional probability of error for the phase estimation. 
The quantity $P_{E}(L ; \phi)$ is of course dependent upon the configuration of the phase estimator itself. The noisy reference problem has been studied in Refs. 1 and 2 for the phase estimators of Figs. $4 \mathrm{a}$ and $4 \mathrm{~b}$. The corresponding expressions for $P_{E}(L ; \phi)$ are respectively given by Eqs. (40) and (19) of the cited references. Assuming that the $M$ carrier tracking loops are of the $L^{\text {th }}$ power loop or L-phase Costas loop types (Ref. 1) and all have identical loop parameters, then the average word error probability of the suboptimum receiver is given by

$$
\begin{aligned}
P_{E} & =\int_{-\pi / L}^{\pi / L} P_{E}(\phi) L p(L \phi) d \phi \\
& =\int_{-\pi}^{\pi} P_{E}\left(\frac{\Phi}{L}\right) p(\Phi) d \Phi
\end{aligned}
$$

In the above, $\Phi \triangleq L \phi$ and $p(L \phi)$ is the p. d. f. of the loop phase error $L \phi(t)$ that can be approximated by

$p(L \phi) \cong \frac{\exp (\beta L \phi+\alpha \cos L \phi)}{4 \pi^{2} \exp (-\pi \beta)\left|I_{j \beta}(\alpha)\right|^{2}} \int_{L \phi}^{L \phi+2 \pi} \exp [-\beta x-\alpha \cos x] d x ;|L \phi| \leq \pi$

where $\alpha$ and $\beta$ are defined in terms of the loop parameters [Eq. (16) of Ref. 1] and $I_{j \beta}(\alpha)$ is the imaginary Bessel function of imaginary order $\beta$ and argument $\alpha$. As an example, consider the case where $N=64$ with $M=32$, $L=2$, and coherent detection of differentially encoded phase symbols (i. e., the phase detector of Fig. 4b). For this case,

$$
P_{E}(\phi)=\operatorname{erfc}\left(\sqrt{R_{b}} \cos \phi\right)\left[1-\frac{1}{2} \operatorname{erfc}\left(\sqrt{R_{b}} \cos \phi\right)\right]
$$

Using (26) as $P_{E}(2 ; \phi)$ in (24), the performance of an L-orthogonal system that employs a Costas loop for tracking and differentially encoded PSK signaling for ambiguity resolution is illustrated in Fig. 7. In this figure, we have assumed a Costas loop filter of the form

$$
F(p)=\frac{1+\tau_{2} p}{1+\tau_{1} p}
$$

and the parameters $\gamma$ and $\rho$ are defined by

$$
\begin{aligned}
& \gamma=\frac{2 W_{L}}{W_{i}} \\
& \delta=\frac{2}{W_{L}^{T}}
\end{aligned}
$$


where $W_{L}$ is the two-sided loop bandwidth and $W_{i} / 2$ is the two-sided bandwidth of the RC low-pass filters in the in-phase and quadrature arms of the Costas loop. Other parameters assumed for the Costas loop are $T_{2} / \tau_{1}=0.002$ and 0.707 damping. The curve marked $\delta=\infty$ corresponds to the case of perfect reference signals.

Conclusions. In conclusion, we have demonstrated several suboptimum receiver structures whose error probability performance is negligibly different from that of the optimum receiver (as predicted by tight upper and lower bounds). For each of these suboptimum receiver structures, we have assessed the tradeoffs between word error performance and bandwidth occupancy as the composition of the signal is varied between the extremes of one frequency with $\mathrm{N}$ phases (polyphase) and one phase with $\mathrm{N}$ frequencies (orthogonal).

In view of the work of Cahn (Ref. 3) and others on the problem of combined digital phase and amplitude modulation, it would be interesting to extend the L-orthogonal signaling technique to the consideration of a combined digital phase, frequency, and amplitude modulation system. The performance tradeoffs obtained would be studied as a function of three degrees of freedom, namely phase, frequency, and amplitude.

\section{References}

1. Lindsey, W. C., Simon, M. K., "Carrier Synchronization and Detection of Polyphase Signals," to appear in IEEE Transactions on Communication Technology, Vol. COM-20, No. 3, June, 1972.

2. Lindsey, W. C., Simon, M. K., "On the Detection of DifferentiallyEncoded Polyphase Signals," presented at the 1972 International Conference on Communications, Philadelphia, Pa.

3. Cahn, C. R., "Combined Digital Phase and Amplitude Modulation Communication Systems," IRE Transactions on Communication Systems, Vol. CS-8, No. 3, pp. 150-154, September, 1960.

4. Golomb, S., et. al., Digital Communications with Space Applications, Prentice Hall, Inc., Englewood Cliffs, N. J., 1964.

5. Stiffler, J. J., Theory of Synchronous Communications, Prentice Hall, Inc., Englewood Cliffs, N. J., 1971.

6. Reed, I. S., Scholtz, R. A., "N-Orthogonal Phase-Modulated Codes," IEEE Transactions on Information Theory, Vol. IT-12, No. 3, pp. 388-395, July, 1966.

7. Viterbi, A., Stiffler, J. J., "Performance of N-Orthogonal Codes," IEEE Transactions on Information Theory, Vol. IT-13, No. 3, pp. 521-522, July, 1967.

8. Nuttall, A. H., "Error Probabilities for Equicorrelated M-ary Signals under Phase-Coherent and Phase-Incoherent Reception," IRE Transactions on Information Theory, Vol. IT -8 , No. 3, pp. 305-314, July, 1962.

9. Fleck, J. T., Trabka, E. A., "Error Probabilities of Multiple-State Differentially Coherent Phase Shift Keyed Systems in the Presence of White, Gaussian Noise," Detect Memo No. 2A in "Investigation of Digital Data Communication Systems," J. G. Lawton, Ed., Cornell Aeronautical Laboratory, Inc., Buffalo, N. Y., Report No. UA-1420S-1, January, 1961 . 


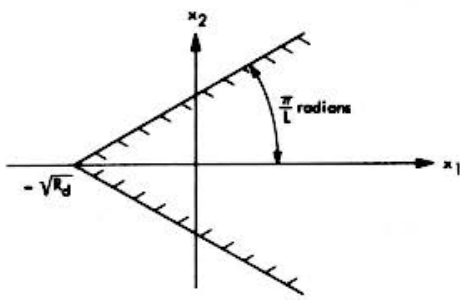

REGION B

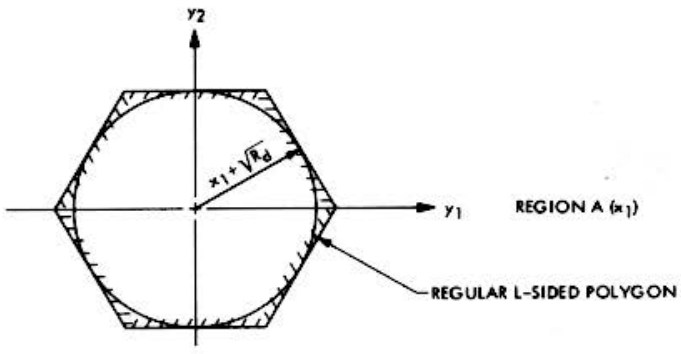

Fig. 1 - Areas of Integration for Equation (11) (Example Drawn for $L=6$ )

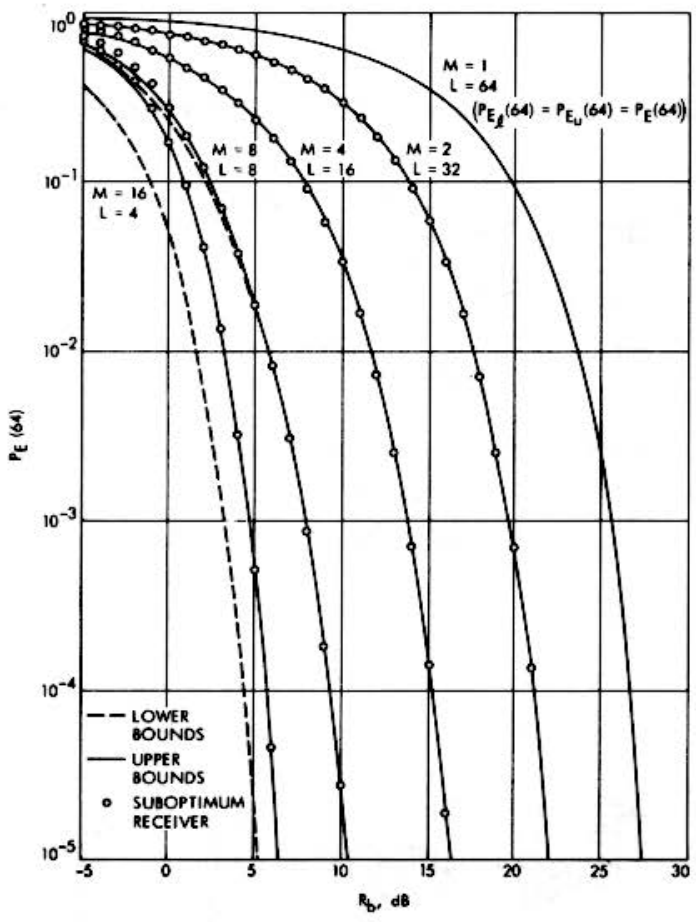

Fig. 2 - Error Probability Performance of L-Orthogonal Signals (Ideal MPSK) 


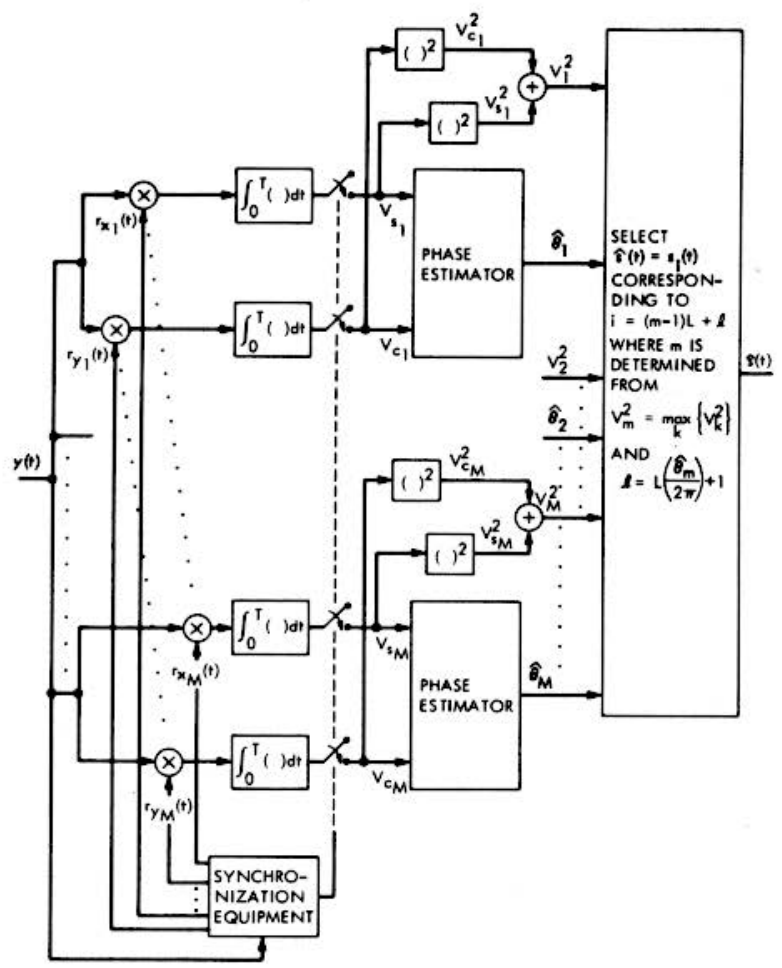

Fig. 3 - A Suboptimal Receiver for Detection of L-Orthogonal Signals (o) MPSK

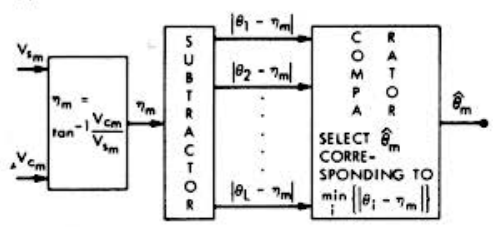

(b) COHERENT DETECTION OF DIFFERENTIALLY ENCODEO MPSK

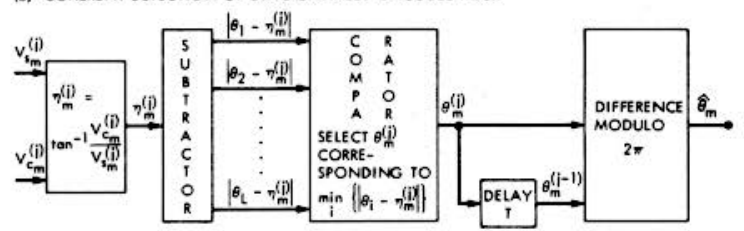

(c) DIFFERENTIALLY COHERENT DETECTION OF DIFFERENTIALLY ENCODED MPSK

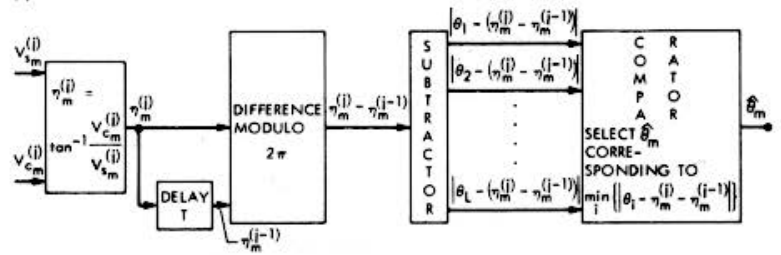

Fig. 4 - The $\mathrm{m}^{\text {th }}$ Phase Estimator Element of the Suboptimal Receiver 


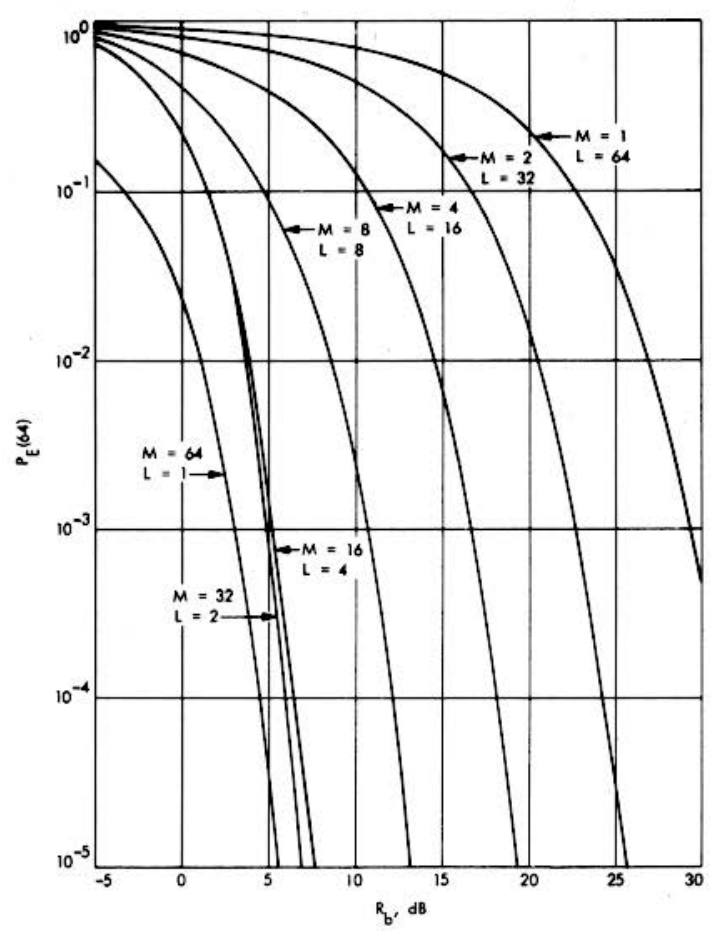

Fig. 5 - Error Probability Performance of L-Orthogonal Signals (Coherent Detection of Differentially Encoded MPSK)

Fig. 6 - Error Probability Performance of L-Orthogonal Signals (Differentially Coherent Detection of Differentially Encoded MPSK)

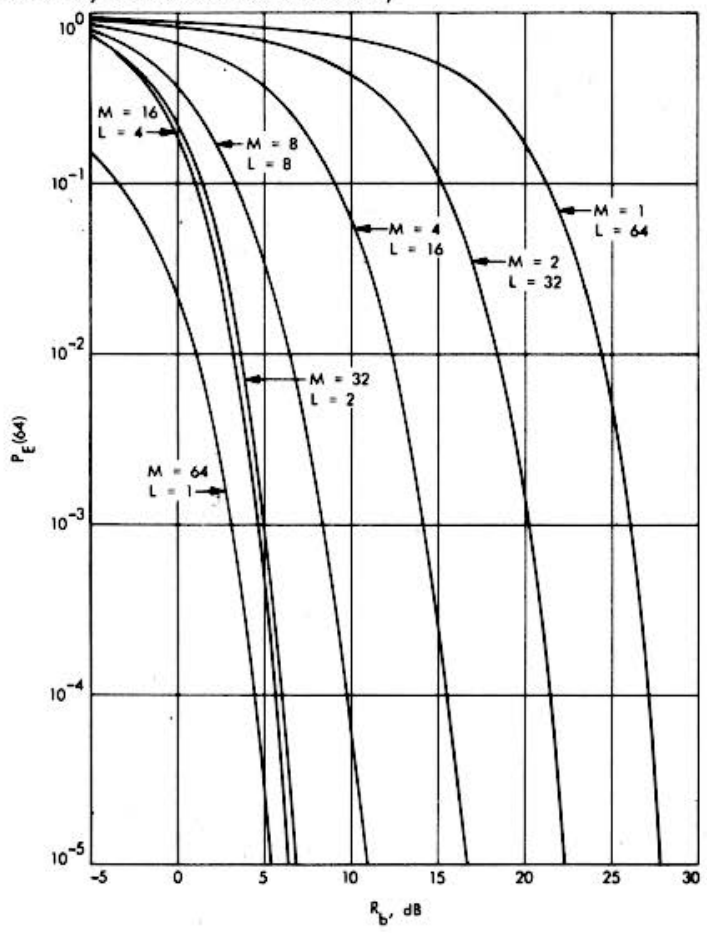




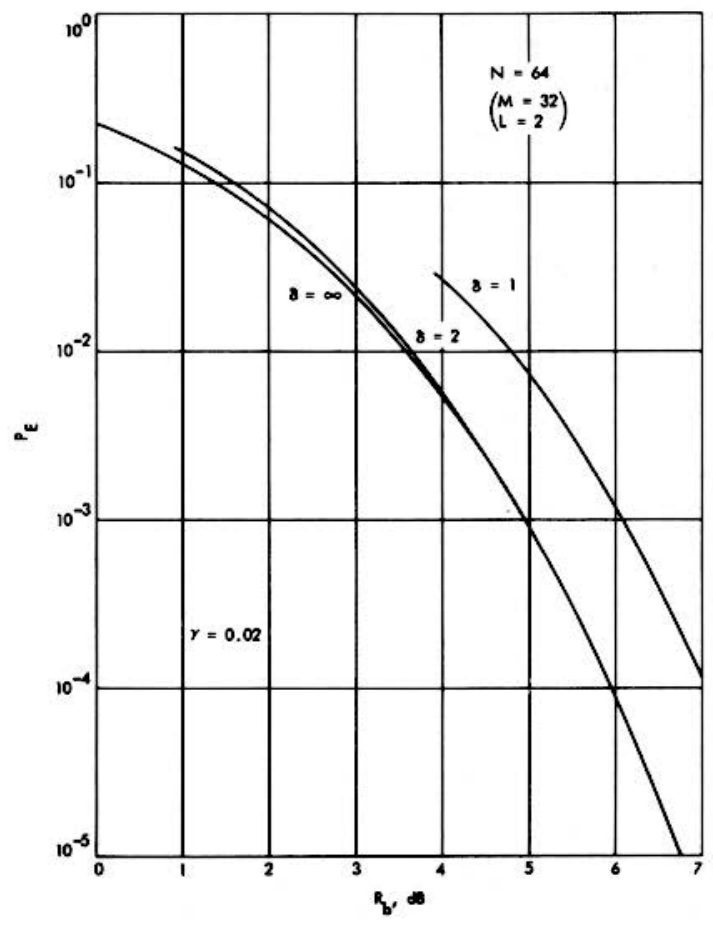

Fig. 7 - Error Probability Performance of L-Orthogonal Signals Where the Noisy Reference Signals are Provided by a Costas Loop

This paper presents the results of one phase of research carried out at the Jet Propulsion Laboratory, California Institute of Technology, under Contract No. NAS 7-100, sponsored by the National Aeronautics and Space Administration. The work was also supported by the Department of the Navy, Office of Naval Research, under Contract NR042-275. 